University of Wollongong

Research Online

Faculty of Informatics - Papers (Archive)

Faculty of Engineering and Information

Sciences

30-10-2006

\title{
A variable length linear array for smart antenna systems using partial optimization
}

W. Raad

University of Wollongong, walid@uow.edu.au

Ian S. Burnett

University of Wollongong, ianb@uow.edu.au

Follow this and additional works at: https://ro.uow.edu.au/infopapers

Part of the Physical Sciences and Mathematics Commons

\section{Recommended Citation}

Raad, W. and Burnett, lan S.: A variable length linear array for smart antenna systems using partial optimization 2006.

https://ro.uow.edu.au/infopapers/537

Research Online is the open access institutional repository for the University of Wollongong. For further information contact the UOW Library: research-pubs@uow.edu.au 


\title{
A variable length linear array for smart antenna systems using partial optimization
}

\author{
Abstract \\ Adaptive arrays have been used extensively in wireless communications applications to reduce \\ interference between desired users and interfering signals. Significant research has been directed at the \\ uniform linear array (ULA), however, generally a fixed array length is used. In a mobile environment this \\ has the disadvantage of producing a fixed beamwidth due to the fixed array lengths. The variable linear \\ array (VLA) algorithm is a proposed algorithm that allows the beamwidth to be varied by activating and \\ deactivating elements depending on the interferers present. This approach uses the LMS algorithm for \\ adaptation, in an embedded system this could prove costly. This paper proposes an alternative approach \\ based on partial optimization techniques that remove the LMS from the optimization process. \\ Furthermore studies into the cost of having such a system is presented and compared to the operational \\ cost of the LMS algorithm.

\section{Disciplines} \\ Physical Sciences and Mathematics

\section{Publication Details} \\ This paper was originally published as: Raad, W \& Burnett, IS, A variable length linear array for smart \\ antenna systems using partial optimization, 10th IEEE Singapore International Conference on \\ Communication Systems 2006 (ICCS 2006), Singapore, 30 October-2 November 2006, 1-5. Copyright IEEE \\ 2006.
}




\section{A VARIABLE LENGTH LINEAR ARRAY FOR SMART ANTENNA SYSTEMS USING PARTIAL OPTIMIZATION}

\author{
Walid Raad \\ School of Electrical, Computer and Telecommunication \\ Engineering, University of Wollongong \\ Wollongong, Australia.
}

\author{
Ian S. Burnett \\ School of Electrical, Computer and Telecommunication \\ Engineering, University of Wollongong \\ Wollongong, Australia
}

\section{ABSTRACT}

\begin{abstract}
Adaptive Arrays have been used extensively in wireless communications applications to reduce interference between desired users and interfering signals. Significant research has been directed at the Uniform Linear Array (ULA), however, generally a fixed array length is used. In a mobile environment this has the disadvantage of producing a fixed beamwidth due to the fixed array lengths. The Variable Linear Array (VLA) algorithm is a proposed algorithm that allows the beamwidth to be varied by activating and deactivating elements depending on the interferers present. This approach uses the LMS algorithm for adaptation, in an embedded system this could prove costly. This paper proposes an alternative approach based on partial optimization techniques that remove the LMS from the optimization process. Furthermore studies into the cost of having such a system is presented and compared to the operational cost of the LMS algorithm.
\end{abstract}

Keywords- Adaptive Antenna, LMS, Linear Array.

\section{INTRODUCTION}

Adaptive Antenna systems are comprised of two components, the antenna array which is used for transmission and reception purposes and the adaptive algorithm which is used to determine the weight coefficients for optimization of the array. The antenna array can be designed so that the radiation pattern formed contains a main lobe with a specific beamwidth. The adaptive algorithm is used to ensure that the array maintains directivity to a desired user and steers clear of interfering signals. The adaptive algorithm will use information such Angle of Arrival (AOA) and angle spread or angle difference as a means of determining the optimal solution for different situations [1] [2] [3].

A recent trend is the study of variable length arrays, whereby subsets of fixed arrays are selected and optimised. This approach offers advantages such as a reduction in computational complexity, and gives the flexibility required to deal with a changing mobile environment. One such example is the work proposed by Rezk et al [4]. In this instance the authors propose a hybrid smart antenna system that utilises the signal strength as a means of selecting a sub-array for optimization. Another algorithm that has been presented is the Variable Linear Array (VLA) algorithm [5]. This proposal controls the beamwidth of a smart antenna system by varying the number of elements activated when the radiation pattern is produced. This allows the rejection of interfering signals which may affect the overall system performance of the adaptive antenna. The VLA alters the number of radiating elements depending on the determined angle difference between the desired signal and the closest interfering signal. The approach in which the VLA optimises the weight coefficients is by using the LMS algorithm, for an embedded system this could prove costly due to the fact that whenever a change in array length occurs, optimization must be carried out to ensure the radiation pattern is accurate.

The present paper proposes an alternative approach to optimising the weight coefficients in the VLA algorithm. This approach uses partial optimization whereby the weight coefficients of a large array are applied to a smaller array. This approach is incorporated into the VLA algorithm in the form of a lookup table. The cost of the lookup table is studied and a comparison between the original weight coefficients and a range of quantised weight coefficients is presented.

\section{THEORY}

Linear arrays consist of radiating elements spaced in a straight line [6]. With this configuration the linear array provides one dimension of control, whereby the ability to sweep in either the azimuth $(\varphi)$ plane or the adjustment in the elevation $(\theta)$ plane is available but sweeping in both directions simultaneously is not. The radiation pattern of the array is a weighted sum of radiating element patterns and its directivity is achieved by changing the weight coefficients that are calculated using an adaptive algorithm.

Weight coefficients are updated using an adaptive algorithm and for the given weights the radiation pattern is formed based on the following formula [7].

$$
z(t)=A s(t) \sum_{m=0}^{M-1} w_{m} e^{-j \beta \Delta x \cos \phi \sin \theta}=A s(t) f(\theta, \phi)
$$

where $f(\theta, \varphi)$ is the array factor and represents the ratio of the received signal available at the array output, $z(t)$ to the signal $A s(t), w_{m}$ are the weight coefficients for a particular antenna element: $i=1, \ldots, M, \beta=2 \pi / \lambda$ is the phase propagation factor and a pair $(\varphi, \theta)$ denotes the desired angle of arrival.

The beamwidth of an antenna array is the measured angle that the main lobe of the radiation pattern induces. Generally it is measured at the $3 \mathrm{~dB}$ point and the First Null beamwidth (FNBW). The process by which the 
FNBW is obtained when operating a uniformly spaced linear array with the LMS algorithm as the adaptive algorithm can be represented by the following [5]:

$$
\begin{aligned}
& F N B W=2\left[f\left(\theta, \phi_{0}\right)-f\left(\theta, \phi_{2}\right)\right] \\
& F N B W=2\left(\phi_{0}-\phi_{2}\right)
\end{aligned}
$$

Equation 2 represents the FNBW which was used for an optimised linear array for an AOA of $\varphi_{0}$. Where $\varphi_{0}$ is located at the maximum position and the first null is located at $\varphi_{1}$ and $\varphi_{2}$. Furthermore the $3 \mathrm{~dB}$ beamwidth can also be obtained by setting (1) to 0.707 and obtaining the corresponding angles.

The concept of varying the array length is a recent trend that is used to reduce the computation required for optimization. This approach has the advantage of providing greater flexibility for the system to better suit the surrounding wireless environment. By activating and deactivating elements in the array, the beamwidth is effectively altered from being broad (small array) to thin (large array). Rezk [4] proposes such a method and uses the signal strength of each element as the selection criterion. In doing so it is ensured that only the elements with the strongest signal are selected. This selection process is suitable but raises a question regarding the effect of altering the element spacing in a linear array with regards to the radiation pattern and it characteristics such as the beamwidth. As an incoming wave strikes each element at a particular instant in time a phase is generated that is dependent on the wavelength $\lambda(m)$ and element spacing $d(\lambda)$ this can be represented by (4) [8]:

$$
\phi=\frac{2 \pi d \cos \theta}{\lambda}
$$

Equation 4 represents the phase that is generated when the incoming signal strikes the array. The following figure represents the effect of a changing $d$ with regard to the beamwidth for an $\mathrm{AOA}$ of $60^{\circ}$.

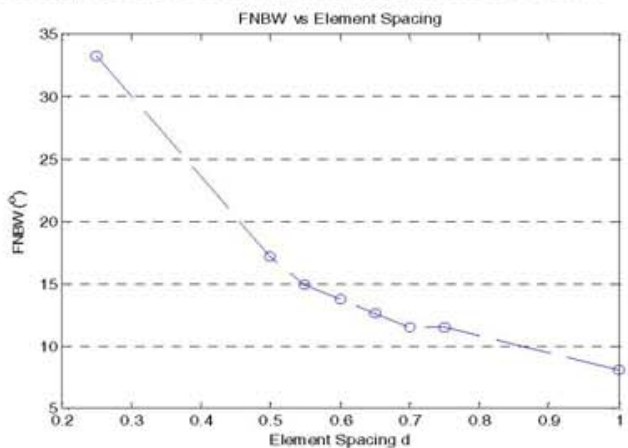

Figure 1: FNBW versus Element Spacing.

It can be seen from figure 1 that a change in element space can produce a change in beamwidth. However characteristics such as sidelobes and grating lobes are not taken into consideration. For example grating lobes will become prominent when the element spacing is large enough for the incoming waves to add in phase [7], for the example above it was observed at $0.65 \lambda$.

In this instance the measurements were obtained for an angle of arrival of $60^{\circ}$ utilising the Least Mean Square
(LMS) algorithm for optimization, which is represented by the following:

$$
\begin{aligned}
& \varepsilon_{k}=d_{k}-X_{k}^{T} W_{k} \\
& W_{k+1}=W_{k}+2 \mu \varepsilon_{k} X_{k}
\end{aligned}
$$

In (5) $\varepsilon$ is the error and represents the difference between the desired and the received signal, $d$ is the desired signal, and $\mu$ is the step size [9] [10] [11].

\section{Partial Optimization for SMart Antenna}

Partial optimization for smart antenna systems is when weight coefficients are calculated utilising an adaptive algorithm such as the LMS algorithm but the optimal values are not obtained. This is generally carried out to reduce the optimization time in the adaptive algorithm [15]. This section describes a proposed method of partial optimization that utilises the calculated weights for larger arrays and applies them to sub-arrays of the same arrival angle. In doing so it removes the process of having to recalculate weight coefficients for every single change in the sub-array size. This process is very beneficial for algorithms such as the VLA when applied to stationary users and the interferers are mobile around the user.

The proposed approach relies on the fact that desired users are stationary in nature, similar to systems such as fixed Broadband Wireless Access (BWA) systems. Experiments conducted show that it is feasible to apply the weights of large arrays to sub-arrays based on the fact that the AOA remains unchanged in the two cases and that the application of weight coefficients is sequential in nature, such that the weight calculated for element No. 1 is applied to the sub-array element No. 1. This ensures that the optimised state for the larger array is maintained in the sub-array, this can be observed in the example shown in Figure 3.

In this instance a maximum of $N=15$ weight coefficients were calculated. From this a subset of $M=8$ was selected. It can be observed that when compared to a fully optimised $M=8$ using the LMS algorithm the radiation parameters such as beamwidth and sidelobes remain unchanged besides being reduced in magnitude.

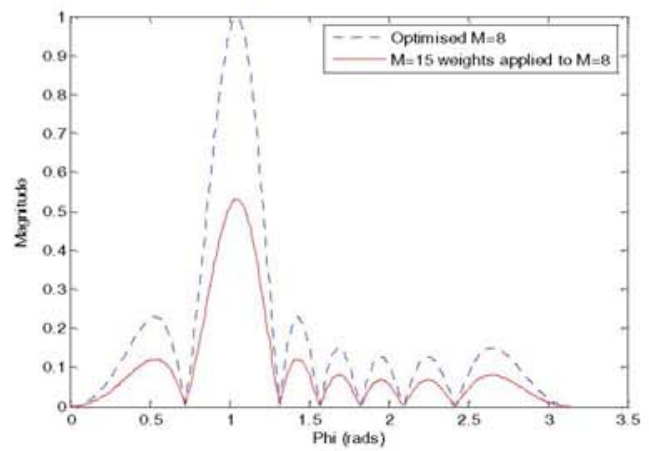

Figure 2: Partial Optimization Technique for an 8element linear Array using weights from $N=15$.

The magnitude of the partially optimised sub-array is reduced due to the fact that the applied weights are 
calculated for a larger array and hence would be smaller in magnitude when optimization occurs. This problem can be easily overcome by applying a compensator that matches the original sub-array weights to that of an optimised array of the same size. This compensator can be represented by the following;

$$
\left(\frac{1}{M / N}\right) w_{m}
$$

Equation 7 mathematically represents the compensator, in this instance $N$ is the maximum array length, $M$ is the sub-array length, $w_{m}$ is the weight coefficient. This compensator will increase the magnitude of the weight coefficient to ensure that the radiation pattern characteristics match. For example when this compensator is applied to the example presented in Figure 2 the following result is observed in Figure 3.

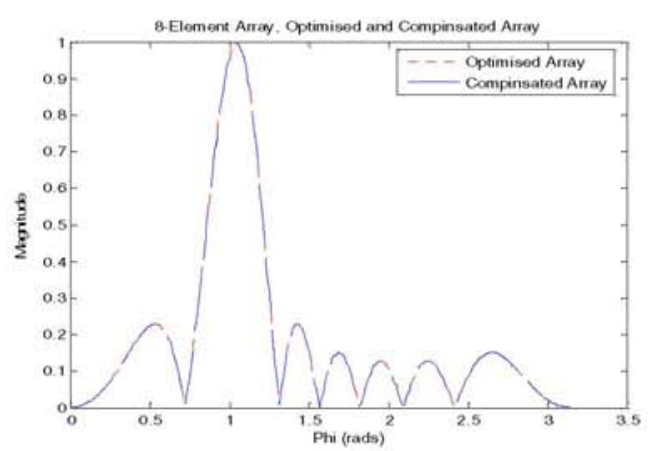

Figure 3: An optimised 8-Elment Linear array versus $M=8$ elements with the compensator applied.

It can be observed that if the conditions mentioned earlier are maintained then the compensator will increases the magnitude of the sub-array weights to match the weights of a fully optimised linear array using the LMS algorithm for adaptation.

\section{The VLA Algorithm Using Partial OPTIMSATION}

The VLA algorithm alters the beamwidth of an array by sequentially activating and deactivating the elements required depending on the surrounding environment. The original method [5] utilised the LMS algorithm to calculate the weight coefficients when the sub-array size was altered. This method can become expensive due to the fact that it has to recalculate the weights every time a change in the array size occurs. The modified VLA utilises the partial optimization procedure discussed in section 3 to remove the optimization process. In doing so this approach reduces the optimization time greatly. This method is beneficial for users that remain still for extended periods such as BWA systems. Hence modifications were introduced on the original systems based on the proposed partial optimization technique mentioned in the previous section.
The proposed method relies on the use of lookup tables containing pre-determined weight coefficients. The design of the lookup table is novel in structure as it is comprised of a set of arrival angles with their corresponding weight coefficients. Based on previous experimentation [5] it was determined that a maximum array size of $N=30$ elements would be used in the lookup table design due to the fact the change in beamwidth is minimal if the array was increased greater than 30 elements. From this a sub-array of $M$-elements would be selected based on information received from the VLA algorithm. Utilising this information a small search is conducted to obtain the closest value available in the lookup table. For this design it was determined that increments of $4^{\circ}$ would be suitable, this is due to the fact that at half-wavelength spacing the limitation with which the VLA can operate is $4^{\circ}$ [5]. Furthermore a sweep capability of $20^{\circ}$ to $90^{\circ}$ was set when determining how entries the lookup table should contain; this limit was set based on the lookup table index.

The VLA algorithm operates on the following assumptions:

- The arrival angles of all incoming signals have been determined and are known to the system. For example in fixed BWA systems subscriber information can be used to determine the $\mathrm{AOA}$ with respect to the base station.

- The desired incoming signal has been determined and is set as the first signal so as to become the reference signal to all other incoming arrival angles.

- The remaining signals are signal copies of the original desired signal.

- The elements in the array are activated sequentially. This is to ensure that radiation pattern characteristics are kept the same as a fully optimised array of the same size.

The VLA algorithm operates firstly by obtaining a number of incoming AOAs;

$\varphi_{n}=\left|\varphi_{1}, \varphi_{2}, \ldots, \varphi_{N}\right|$

where

$n=1,2,3, \ldots, N$ and $\varphi_{1}=\varphi_{\text {desired }}$

The difference is then determined between the desired $\mathrm{AOA}$ and the remaining incoming signals to produce a vector of angle differences.

$$
\varphi_{\text {diff }}=\left|\varphi_{\text {diff } 1}, \varphi_{\text {diff } 2}, \ldots, \varphi_{\text {diffN }}\right|
$$

Equation 9 is the resultant angle differences between the desired signal and the remaining incoming signal AOA. The smallest difference in (9) is then used to select the number of elements in association with the desired AOA.

$$
\begin{aligned}
& \underset{\phi_{\text {smallet }}}{\arg \min }=\left|\phi_{\text {diff }}\right| \\
& B W_{\text {required }}=2 \varphi_{\text {smallest }}
\end{aligned}
$$


Equation 11 multiplies the smallest difference to ensure that the both sides of the main radiation lobe are incorporated.

The process of how the lookup table is selected is based on the following approach; each value in the vector represents a particular lookup table.

$$
\varphi_{\text {lookUpInde } x}=|0,30,45,60,90|
$$

By utilising the desired $\mathrm{AOA}$ and equation 12 the closest lookup table is sought according to the minimisation of:

$$
\begin{aligned}
& \left|\varphi_{\text {lookUpDiff }}\right|=\varphi_{\text {desired }}-\varphi_{\text {lookUpInde }} x \\
& \underset{\phi_{\min \text { diff }}}{\arg \min }\left|\varphi_{\text {lookUpDiff }}\right|
\end{aligned}
$$

Equation 13 produces a vector of differences between the desired AOA and the lookup index vector. The minimum value is obtained and the lookup table to select is determined by:

$$
\varphi_{\min \text { diff }}=\varphi_{\text {lookUpDiff }}
$$

In (15) the lookup table is determined by searching $\varphi_{\text {lookUpDiff }}$ and comparing $\varphi_{\text {mindiff }}$ when the value is obtained the location of the value is associated to the required lookup table as defined in (12). From this the number of elements to achieve the required beamwidth is selected and passed to the partial optimization table. Firstly a vector index of corresponding arrival angles is defined in the following manner:

$$
\begin{aligned}
& \mid \text { Index }|=| A O A_{1}, A O A_{2}, \ldots \ldots \ldots . ., A O A_{N} \mid \\
& \text { where } N=20,24,28, \ldots \ldots \ldots ., 112
\end{aligned}
$$

The next step in the process is to subtract the Index vector from the desired arrival angle $\varphi_{\text {desired }}$. This forms a new vector of values to determine the closest value present in the lookup table.

$$
\begin{aligned}
& |R|=\varphi_{\text {desired }}-\text { Index } \\
& \underset{\phi_{\min \text { made }}}{\arg \min }|R|
\end{aligned}
$$

Equation 17 and 18 determine the required row that needs to be selected to obtain the correct weight coefficients. Once the weight coefficients have been selected the weight compensator is applied as defined in Equation 7. Hence the partially optimised weights are then applied to produce the radiation pattern in (1).

\section{RESULTS}

The partial optimization technique applied to the VLA algorithm was compared in terms of complexity to the VLA algorithm utilising a standard LMS algorithm. As seen in equations (5) and (6) the LMS algorithm operates on an iterative approach to optimise the required weight coefficients. Therefore the cost of the LMS algorithm per element is equal to 4 multiplications and 2 additions; this is then multiplied by the total number of iterations the LMS requires to optimise the weight coefficients.

For example an 8-element linear array using the LMS algorithm with 150 iterations as was the case with the simulations conducted will require at total of 4800 multiplications and 2400 additions.

The partial optimization technique, on the other hand, only requires one addition to determine the required row in the table, then a search and compare is carried out to retrieve the required weight coefficients, and finally just 1 multiplication to compensate the selected weights. For the proposed lookup table there are 18 entires, and the total cost of executing such a process would be 19 operations, whereby 18 additions are used for the sequential search and 1 multiplication for the compensator. By removing the continual optimization process of the LMS algorithm considerable reductions in complexity can thus be achieved.

The cost of the lookup table can be considered further by analysing the memory cost of the weight coefficients in an embedded system. For example, the lookup table used in this simulation had a sweep capability of $20^{\circ}$ to $90^{\circ}$; this corresponds to a total of 18 rows, with each row containing 30 elements, hence giving a total table size of 540 individual complex weight coefficients requiring storage of 1080 double floating point values. Based on this information, and the fact that MATLAB utilises 64-bits [14] to represent a value of type double, the total storage requirement is $69.12 \mathrm{~kb}$ or $8.64 \mathrm{kB}$. Our investigations have shown that a more coarse quantisation of the weight coefficients reduces the total size of the lookup table without affecting the overall radiation pattern performance. It was determined that the

(16) weight coefficients can be represented by a 6 -bit value without affecting the overall performance of the radiation pattern, for example introducing unwanted sidelobes and grating lobes. In this sense the entire lookup table mentioned above can be stored in a total of 810 Bytes. Figure 4 is the radiation pattern when using 6-bit weight coefficients in comparison to 16-bit weight coefficients for a 30 element linear array with an $\mathrm{AOA}$ of $60^{\circ}$. The error that is generated between the two patterns is also shown.

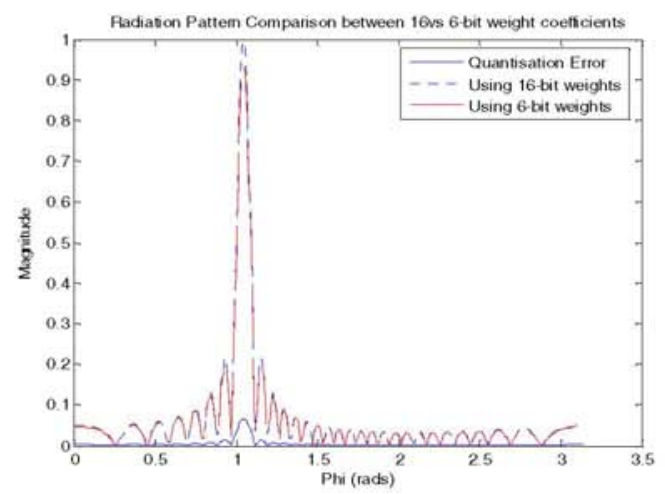

Figure 4: Radiation Pattern Comparison between using 16-bit and 6-bit representation.

It can be observed that the 6-bit quantised weight coefficients produce an accurate radiation pattern output 
whereby the pattern characteristics such as beamwidth, accuracy and sidelobes are similar with that of a radiation pattern using 16-bit weight coefficients. From Figure 4 it can be seen that the error that is generated by the 6-bit quantised weight coefficients is small in comparison to the 16-bit radiation pattern, in this instance it occupies less than $6.5 \%$. Coarse quantisation of the weight coefficients results in a reduction in the total power used by the system when storing and retrieving the lookup table. Furthermore by reducing the storage requirements of the individual values the lookup table is able to store more values and hence offer a larger sweep range.

\section{CONCLUSION}

This paper presented an alternative approach for the VLA algorithm using a novel partial optimization technique that utilised weight coefficients for larger arrays and applied the weights to smaller sub-arrays. It was observed that this technique proved effective with the aid of a compensator which amplified the magnitude to suit the sub-array. Finally the proposed technique was applied to the VLA algorithm and compared to the VLA algorithm when using the LMS for optimization. It was observed that a reduction in cost when operating the lookup was achieved. Furthermore the lookup table approach was analysed from an embedded system approach. in doing so it was observed that by quantising weight coefficients a considerable reduction in the size of the lookup was obtained.

\section{ACKNOWLEDGMENT}

I would like to thank Dr Mohommed Raad and Ibrahim Raad for their support in writing this paper. Also a thankyou must go to Argus Technologies Pty Ltd for their continued support of this project.

\section{REFERENCES}

[1]. W. Cao, W. Wang, "Effects of Angular Spread on Smart Antenna Systems with Uniformly Linear Antenna Array", $10^{\text {th }}$ Asia-Pacific Conf on Communications and $5^{\text {th }}$ International Symposium on Multidimensional Comms, IEEE, 2004

[2]. A. M. Rao, D. L. Jones, "Efficient Detection with Arrays in the Presence of Angular Spreading", IEEE Trans on Signal Processing, Vol. 51, No. 2, pp 301-312, Feb 2003.
[3]. I. Jami, R. F. Ormondroyd, E. Artarit, "Improved Handset Tracking using Kalman Filter Algorithm Aided by Angle Spread Information from a Smart Antenna Array", $58^{\text {th }}$ Vehicular Technology Conference IEEE, Vol. 2, pp 752756, Oct 6-9, 2003.

[4]. M. Rezk, W. Kim, Z. Yun, M. F. Iskander, "Performance Comparison of a Novel Hydrid Smart Antenna System versus the Fully Adaptive and Switched Beam Antenna Arrays", IEEE Antennas and Wireless Prop Letters, Vol. 4, pp 285-288, 2005.

[5]. W. Raad, I. S. Burnett, I. S. Raad, "A Variable Length Linear Array for Smart Antenna Systems", IEEE Int Conf on Information and Communication Technology, April 24 $28,2006$.

[6]. W. H. Kummer, "Basic Array Theory", Proceedings of IEEE, Vol. 80, No. 1, pp. 127-140, 1992.

[7]. J. C. Liberti, T. S. Rappaport, Smart Antennas for Wireless Communications: IS-95 and Third Generation CDMA Applications, 1999.

[8]. H. Acheichi, Implementation Of An Adaptive Antenna System-Undergraduate Thesis, University of Wollongong, 2004.

[9]. R.C.Hansen, Phased Array Antennas, John Wiley \& Sons, New York, 2001.

[10].L. C. Godara, "Applications of Antenna Arrays to Mobile Communications, Part II: Beam-forming and Direction-ofArrival Considerations", IEEE Proceedings, Vol. 85, No. 8, pp 1195-1245, Aug 1997.

[11].M. Rezk, W. Kim, Y. Zhengqing, M. F. Iskander, "Narrow beam adaptive array for advanced wireless applications", IEEE/ACES, Wireless Communications and Applied Computational Electro magnetics, pp 594 - 597, 3-7 April 2005

[12].M .K. Ragheb, S. H. Elramly, S. Mostafa, "DOA estimation using the LMS and the CMA algorithms in a macrocell environment modeled by the GBSBCM", IEEE Proceedings of the Twenty-First National Radio Science Conference, pp C4 - 1-11, 16-18 March 2004.

[13].F. Babich, M. Comisso, M. D'Orlando, L. Mania, "Performance Evaluation of MANETs Using Smart Antennas in Multipath Fading Environment”, $2^{\text {nd }}$ IEEE conf International Symposium on Wireless Communication Systems, pp 327 - 331, 05-09 Sept. 2005.

[14].MATLAB version7 help files.

[15].V. H. Nascimento, "Improving The Initial Convergence of Adaptive Filters: Variable-Length LMS Algorithms", $14^{\text {th }}$ International Conf on Digital Signal Processing, IEEE, Vol. 2, pp667-670, July 1-3, 2002. 